\title{
Metagenomic analysis of captive Amur tiger faecal microbiome
}

\author{
Fengping $\mathrm{He}^{1}$, Dan Liư ${ }^{2}$, Le Zhang ${ }^{1}$, Jiancheng Zhai ${ }^{1}$, Yue Ma ${ }^{1,3}$, Yanchun Xu ${ }^{1,3}$, Guangshun Jiang ${ }^{1,4}$, \\ Ke Rong ${ }^{1,4^{*}+}$ and Jianzhang $\mathrm{Ma}^{1,4^{*}+}$ (iD
}

\begin{abstract}
Background: The gastrointestinal tracts of animals are home to large, complex communities of microbes. The compositions of these communities ultimately reflect the coevolution of microorganisms with their animal host and are influenced by the living environment, diet and immune status of the host. Gut microbes have been shown to be important for human disease and health, but little research exists in the gut microbiome of the Amur tiger, which is one of the most endangered species in the world.

Results: In this study, we present the use of whole-metagenome shotgun sequencing to analyze the composition and functional structures of the gut microbiota in captive Amur tigers. Our results showed a high abundance of four major phyla in captive Amur tigers, including Proteobacteria, Firmicutes, Actinobacteria and Fusobacteria. Moreover, at the genus level, Escherichia, Collinsella and Fusobacterium were most abundant in the captive Amur tiger fecal metagenome. At the species level, Escherichia coli, Fusobacterium ulcerans and Fusobacterium varium were the species with highest abundances in the captive Amur tiger gut microbiota. The primary functional categories of the Amur tiger faecal metagenome were associated mainly with Carbohydrate metabolism, Membrane transport and Amino acid metabolism based on the KEGG pathway database. The comparative metagenomic analyses showed that the captive Amur tiger fecal metagenome had a lower abundance of Spirochaetes, Cyanobacteria and Ascomycota than other animals, and the primary functional categories were primarily associated with carbohydrate metabolism subsystems, clustering-based subsystems and protein metabolism.
\end{abstract}

Conclusions: We presented here for the first time the use of the shotgun metagenomic sequencing approach to study the composition and functional structures of the gut microbiota in captive Amur tiger.

Keywords: Gastrointestinal microbiota, Amur tiger, Panthera tigris altaica, Metagenomics, Pyrosequencing

\section{Background}

The Amur tiger (Panthera tigris altaica), also called the Siberian tiger, is the largest extant tiger subspecies in the genus Panthera (Mammalia, Carnivora, Felidae) [1]. Amur tigers, which are found in the Russian Far East [2] and Northeast China [3], are listed as Endangered on the IUCN Red List of Threatened Species ${ }^{\mathrm{Tx}}$ and are included in CITES Appendix I. The population of wild Amur tiger continues to decline due to habitat loss and degradation, poaching and prey depletion [4].

\footnotetext{
* Correspondence: rongke@nefu.edu.cn; jianzhangma@163.com

${ }^{\dagger}$ Ke Rong and Jianzhang Ma contributed equally to this work.

${ }^{1}$ College of Wildlife Resources, Northeast Forestry University, Harbin 150040, China

Full list of author information is available at the end of the article
}

Fortunately, the Amur tiger has been successfully bred in China, and the population of the Amur tiger has increased from 27 to approximately 1000 after more than 30 years of hard work. However, with the growing population of the Amur tiger, veterinary antibiotics and antiparasitics have been widely used to maintain the healthy development of the population in the Heilongjiang Siberian Tiger Park.

Many studies have shown that the gastrointestinal tracts of humans and animals contain large, complex microbial communities [5-7]. The gut microbiota is now recognized as a coevolutionary partner that facilitates host nutritional acquisition, immune modulation, and homeostasis in response to profound lifestyle changes [8-12]. The diversity and function of the gut microbiota may indicate host

(c) The Author(s). 2018 Open Access This article is distributed under the terms of the Creative Commons Attribution 4.0 International License (http://creativecommons.org/licenses/by/4.0/), which permits unrestricted use, distribution, and 
genotypic characteristics and reflect an adaptive ecosystem response to the host diet and living habits.

In recent years, metagenomic sequencing based on next-generation sequencing technologies [13] has been used to describe the microbial diversity and functional capacity of microbial communities in the gastrointestinal tracts of humans [14, 15] as well as some animal species [16-22]. In this study, for the first time, we used whole metagenome shotgun sequencing to profile the microbial flora inhabiting the digestive system of captive Amur tiger and identify the functional attributes encoded in the gut microbiome.

\section{Results}

We sequenced the faecal metagenomes of 3 captive Amur tigers using the Illumina platform and obtained $32.39 \mathrm{~GB}$ of high-quality bases that were free of adaptor and tiger DNA contaminants (Additional file 1). The unique sequence reads that passed the quality control filtering step were then subjected to further analyses focused on biodiversity and functional annotation. The metagenomic sequence data from this study have been submitted to the National Center for Biotechnology Information (NCBI) Sequence Read Archive (SRA) under accession numbers SRP119699.

\section{Phylogenetic analysis of Amur tiger faecal bacteria, eukaryotes, archaea, and viruses}

The phylogenetic computation revealed a profile comprising $98.10 \%$ bacteria, $1.89 \%$ viruses, $0.01 \%$ eukaryotes and $0.003 \%$ archaea. In the Amur tiger intestinal metagenome, Proteobacteria was the most predominant phylum (44.39\%), followed by Firmicutes (31.38\%), Actinobacteria (9.92\%), and Fusobacteria (8.20\%) (Fig. 1).

Of the Proteobacteria, most belonged to Enterobacteriaceae, and a very small amount were Betaproteobacteria or Pseudomonadales (Additional file 2). The dominant family of intestinal microbes in the Amur tiger is Enterobacteriaceae, and most of the Enterobacteriaceae were Escherichia coli. Firmicutes was the second most predominant phylum in captive Amur tiger gastrointestinal tract, with Clostridiales as the primary contributor to the Firmicutes population, followed by Erysipelotrichales and Selenomonadales (Additional file 2). Among Clostridiales, Lachnospiraceae, Peptostreptococcaceae and Clostridiaceae were the families with the highest abundance. A relatively high proportion of the Clostridiales content was attributable to Ruminococcus gnavus, Clostridium hiranonis and Clostridium perfringens. Erysipelotrichales contains a single family, Erysipelotrichaceae, and this family was represented mainly of Erysipelotrichaceae bacterium nk3d112, Solobacterium moorei and Holdemanella biformis. Selenomonadales was mostly represented by Veillonellaceae, including Megamonas funiformis, $M$. rupellensis and $M$. hypermegale.

Similarly, Coriobacteriia was the primary contributor to the population belonging to the phylum Actinobacteria, followed by the class Actinobacteria. The major genus in the Actinobacteria phylum was Collinsella. Collinsella stercoris and $C$. intestinalis were the predominant species

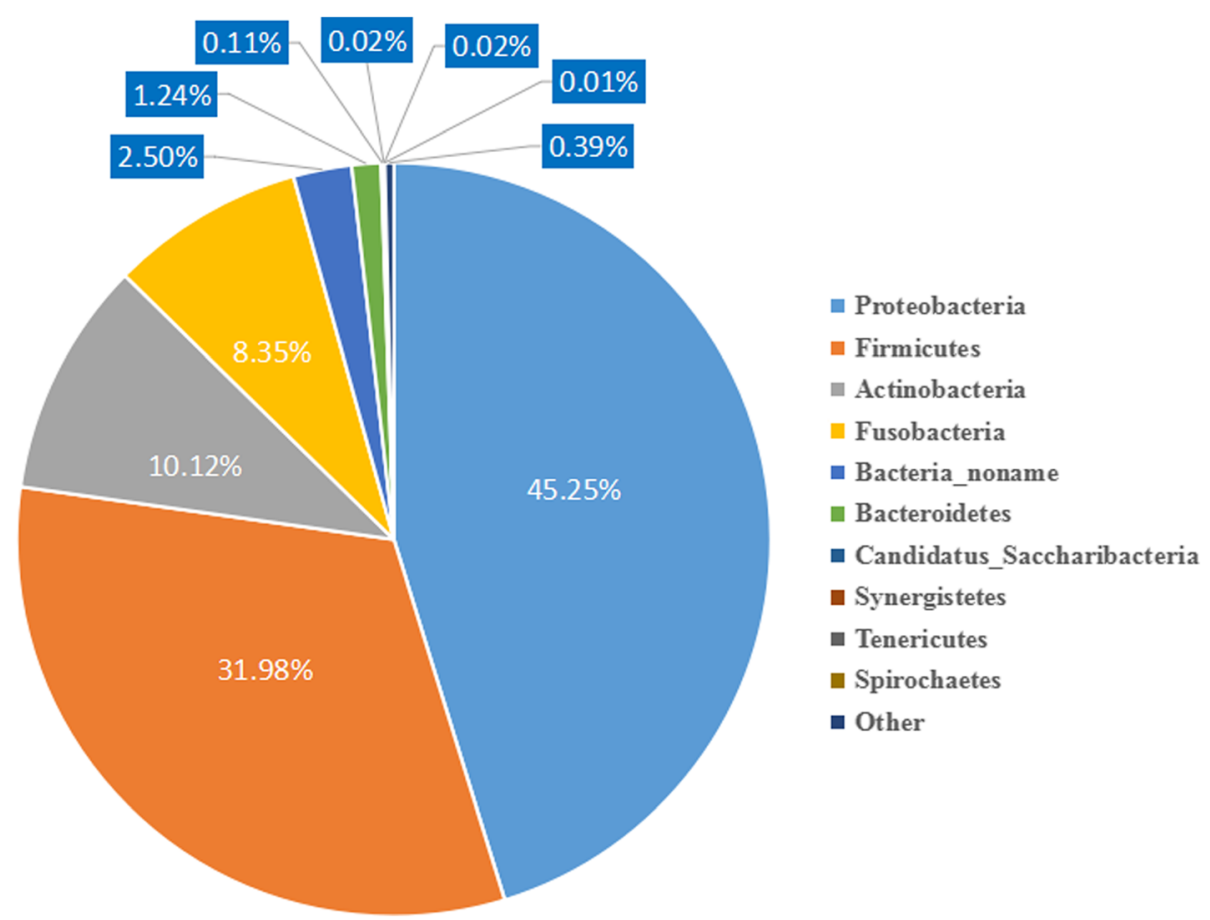

Fig. 1 Bacterial phylum profiles of the Amur tiger microbiome 
among Collinsella in the Amur tiger metagenome. Actinobacteria was also represented by Bifidobacterium, Actinomycetes and Corynebacterium, with each genus represented by a variety of microbial species, such as Actinomyces cardiffensis, Actinomyces turicensis, Corynebacterium diphtheriae and Bifidobacterium breve. The major genus in the Fusobacteria phylum was Fusobacterium. Fusobacterium ulcerans, F. varium, F. mortiferum and $F$. perfoetens were the main species among the Fusobacteria in the Amur tiger metagenome. Fusobacteria are a phylum of bacteria that were significantly more abundant in Carnivora (e.g., Felidae and Canidae) [23]. A high proportion of Fusobacteria were also discovered in the gut microbiomes of some carnivorous vertebrates, such as vultures and alligators [24, 25].

Eukaryota was a minor constituent $(0.01 \%)$ of the Amur tiger metagenome. Most of the Eukaryota were Ascomycota, with a small fraction of Basidiomycota (Additional file 3). Ascomycota is the largest Eukaryota phylum. Trichophyton tonsurans was the primary contributor to the Ascomycota population, followed by Pseudogymnoascus pannorum. Archaea sequences had very low abundance $(0.003 \%)$ in the Amur tiger metagenome, with Euryarchaeota the primary contributor (Additional file 4). In the Amur tiger faecal metagenome,
Methanosarcinaceae was the major Archaea component and included Methanosarcina sp. WH1 and Methanomethylovorans hollandica. Only $1.89 \%$ of the Amur tiger metagenome sequences were viruses, of which Caudovirales was the only order identified (Additional file 5). The major family in Caudovirales was Podoviridae, followed by Siphoviridae and Myoviridae.

\section{Metabolic profiles of the Amur tiger metagenome}

In addition to providing novel insights on the taxonomic diversity of the characteristic microbial consortium, our metagenomic research enabled the identification of the gene functions involved in the symbiotic functions with the host. Based on the KEGG PATHWAY database, hits were classified into 6 groups comprising 42 functional categories (Fig. 2).

Metabolism was the largest group, with higher proportions of Carbohydrate metabolism, Amino acid metabolism, $\mathrm{Nu}$ cleotide metabolism, Metabolism of cofactors and vitamins and Energy metabolism. Collectively, the 12 functional categories in the metabolism group accounted for $86.62 \%$ of the hits. Carbohydrate metabolism and Amino acid metabolism were the most abundant functional categories, representing 16.75 and $11.96 \%$ of the Amur tiger faecal metagenome, respectively. Environmental information processing was the

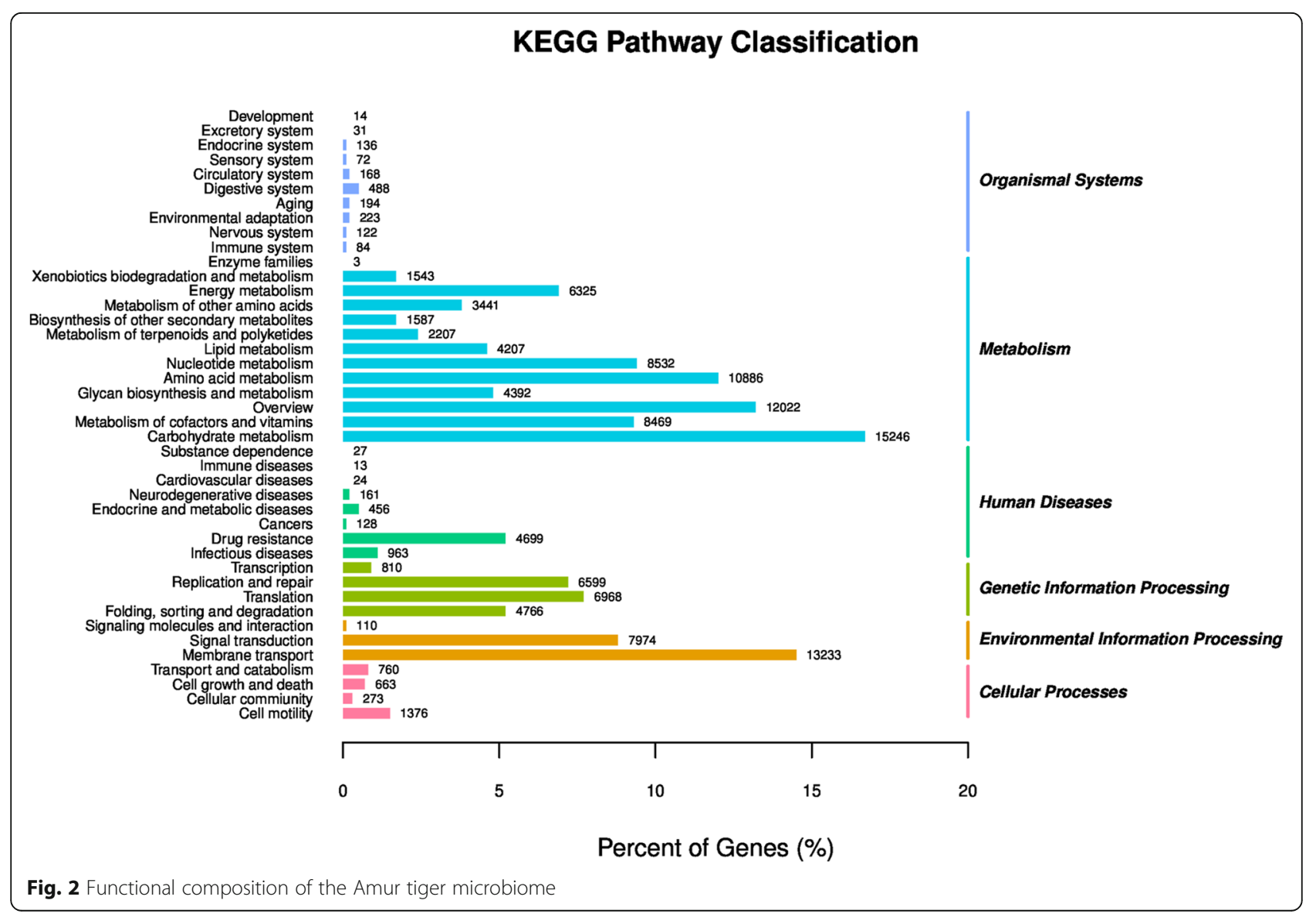


second most prominent functional group, Membrane transport was the most abundant functional categories, followed by Signal transduction and Signalling molecules and interaction. Approximately $14.54 \%$ of the annotated reads from the Amur tiger faecal metagenomes were categorized within Membrane transport.

The third most prominent functional group was Genetic information processing, which included the categories of Translation, Replication and repair, Folding, sorting and degradation. The fourth most prominent group, Human diseases, was dominated by the categories Drug resistance, Endocrine and metabolic diseases and Neurodegenerative diseases. The remaining two groups of captive Amur tiger faecal metagenomes were Cellular processes and Organismal systems. Cell motility, Transport and catabolism and Cell growth and death were important categories within Cellular processes. Most hits of the Organismal system group were distributed among the Digestive system, Environmental adaptation and Aging.

To fully describe the attributes of the genes and gene products identified by faecal metagenome sequencing of the Amur tiger, we performed GO annotations of the unigenes obtained from sequencing. A total of 401,606 unigenes were annotated with $7961 \mathrm{GO}$ functions belonging to molecular function, biological process and cellular components (Additional file 6). The biological processes group contained 157,188 (39.14\%) unigenes that were annotated with $4506 \mathrm{GO}$ functions. The most abundant in the biological process group was biological processes, followed by transport and regulation of transcription, DNA-templated (Additional file 7). In the cellular components group, a total of 76,428 (19.03\%) unigenes were annotated with $851 \mathrm{GO}$ functions, mostly belonging to the cytosol, plasma membrane and cellular components. A total of $167,942(41.82 \%)$ unigenes were annotated with $3051 \mathrm{GO}$ functions in the molecular function group, in which molecular function was the largest category, followed by protein binding and ATP binding.

To further understand the carbohydrate enzymes present in the Amur tiger gut microbiome, we submitted the samples to the Carbohydrate-Active enZYmes database (CAZy). A total of 21,404 annotation results were obtained for the faeces metagenome sequences of the Amur tiger (Additional file 8). Among the 6 large functional CAZy classes, glycosyl transferase (GT) families were the most abundant, followed by glycoside hydrolase $(\mathrm{GH})$ families (Additional file 9). In the further functional subclasses, GT4 and GT2 belonging to the GT family were the most abundant, followed by CBM50 of the CBM family and GH23 and GH13 of the GH family.

\section{Discussion}

In this study, whole-metagenome shotgun sequencing was performed to assess the composition and functional structure of the gut microbiota of the captive Amur tiger. In our study, we observed a high abundance of four major phyla, Proteobacteria, Firmicutes, Actinobacteria and Fusobacteria. Moreover, at the genus level, Escherichia, Collinsella and Fusobacterium were most abundant in the captive Amur tiger fecal metagenome. Importantly, at the species level, Escherichia coli, Fusobacterium ulcerans and Fusobacterium varium were the species with the highest abundances in the captive Amur tiger gut microbiota.

Despite extensive variation among individuals, the gut microbiota of members of the same species are often more similar to one another compared with those of other species. Thus, it is important to provide a comparison between the gut microbiota of the Amur tiger and those of other animals. The results of this study were compared with data sets from different animals, including humans, in the MG-RAST database. Paired data from other studies were chosen, including three wild Amur tigers (WT1, WT2, and WT3), two cats (cat 1 and cat 2), two chickens (chicken 1 and chicken 2), two humans (human 1 and human 2), two mice (mouse 1 and mouse 2), two pandas (panda 1 and panda 2), two pigs (pig 1 and pig 2) and two wolves (wolf 1 and wolf 2 ), which were compared with Amur tigers in captivity (ptg_z1, ptg_z2, and ptg_z3).

The comparisons were performed at the phylogenetic (Fig. 3) and the metabolic levels (Fig. 4). In the phylogenetic comparison, Actinobacteria, Bacteroidetes, Firmicutes and Proteobacteria were the most abundant in all the samples, and the captive Amur tiger samples clustered with the wild Amur tiger samples. Interestingly, the abundance of Apicomplexa and Euryarchaeota were lower in the captive Amur tiger samples than in those from the wild Amur tigers. In addition, the heat map shows that the captive Amur tiger fecal metagenome had a lower abundance of Spirochaetes, Cyanobacteria and Ascomycota than other animals. These findings suggest that the characteristics of gut microbial diversity may adapt to the habits and diet of the Amur tiger in captivity. The metabolic comparison showed that the captive Amur tiger, wild Amur tiger, cat, panda, wolf and human samples clustered together and separated from those of pigs. As expected, all the gut metagenomes were dominated by carbohydrate metabolism subsystems, and the Protein Metabolism and Clustering-based subsystems were represented in relatively high abundance as well.

Intestinal microbial community structure is the result of the coevolution of the microbes and host animal and its environment, and intestinal microorganisms influence physiological function. The intestinal microbial community is also affected by the survival environment and feeding habit of the host animal [26]. Wild felids are susceptible to changes in metabolism and behaviour when 


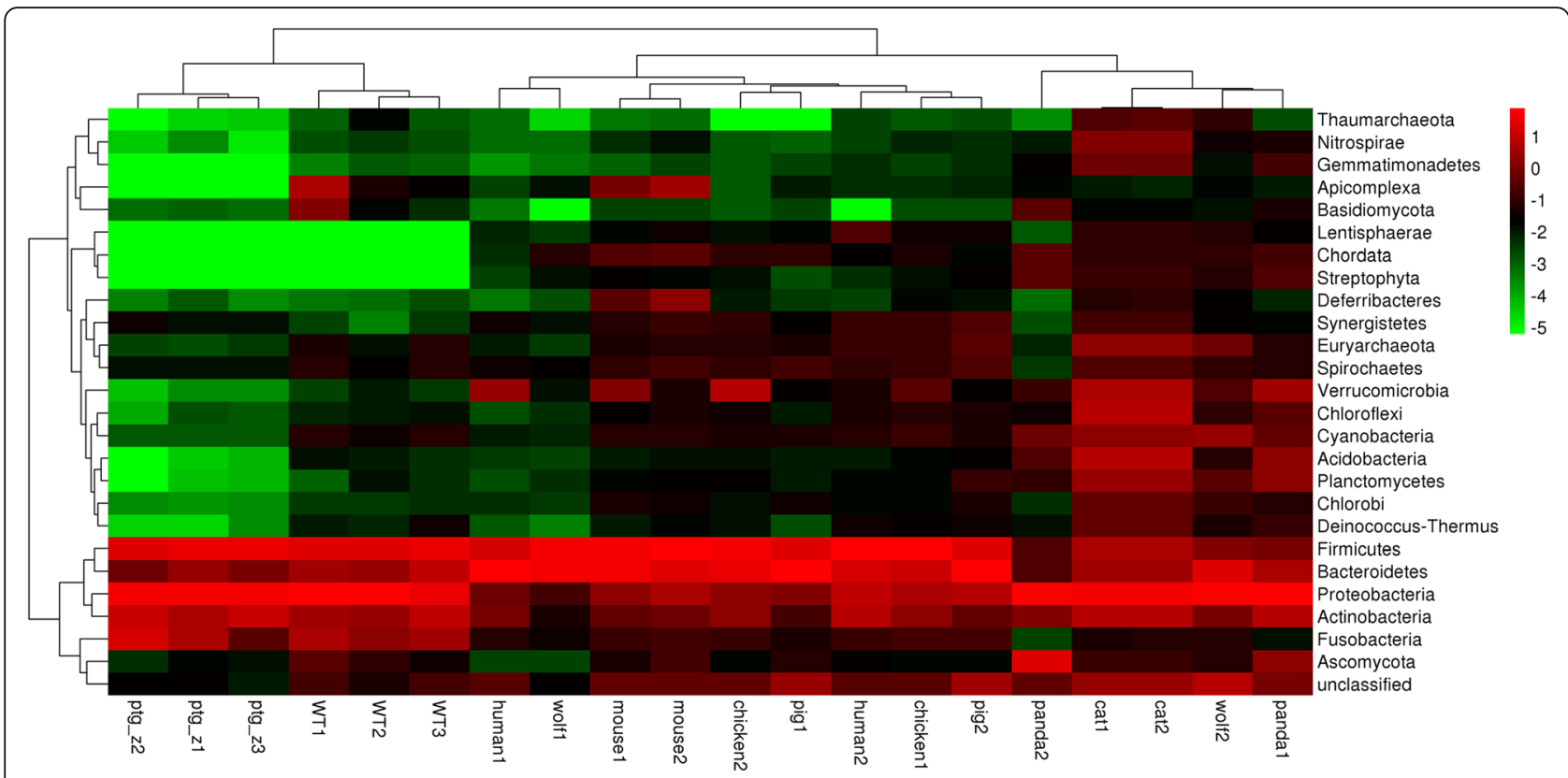

Fig. 3 Heat map of phylogenetic clustering of the wild Amur tiger gut metagenome compared to those of other animals

kept in captivity; these changes are linked to differences from their natural habitat and feeding patterns [27-31].

\section{Conclusions}

In this study, we present the use of whole-metagenome shotgun sequencing to analyze the composition and functional structures of the gut microbiota in captive Amur tigers. Our results showed a high abundance of four major phyla in captive Amur tigers, including Proteobacteria, Firmicutes, Actinobacteria and Fusobacteria. Moreover, at the genus level, Escherichia, Collinsella and Fusobacterium were most abundant in the captive Amur tiger fecal metagenome. At the species level, Escherichia coli, Fusobacterium ulcerans and Fusobacterium varium were the species with highest abundances in the captive Amur tiger gut microbiota. The primary functional categories of the Amur tiger faecal metagenome were associated mainly with Carbohydrate metabolism, Membrane transport and Amino acid metabolism based on the KEGG pathway database. The comparative metagenomic analyses showed

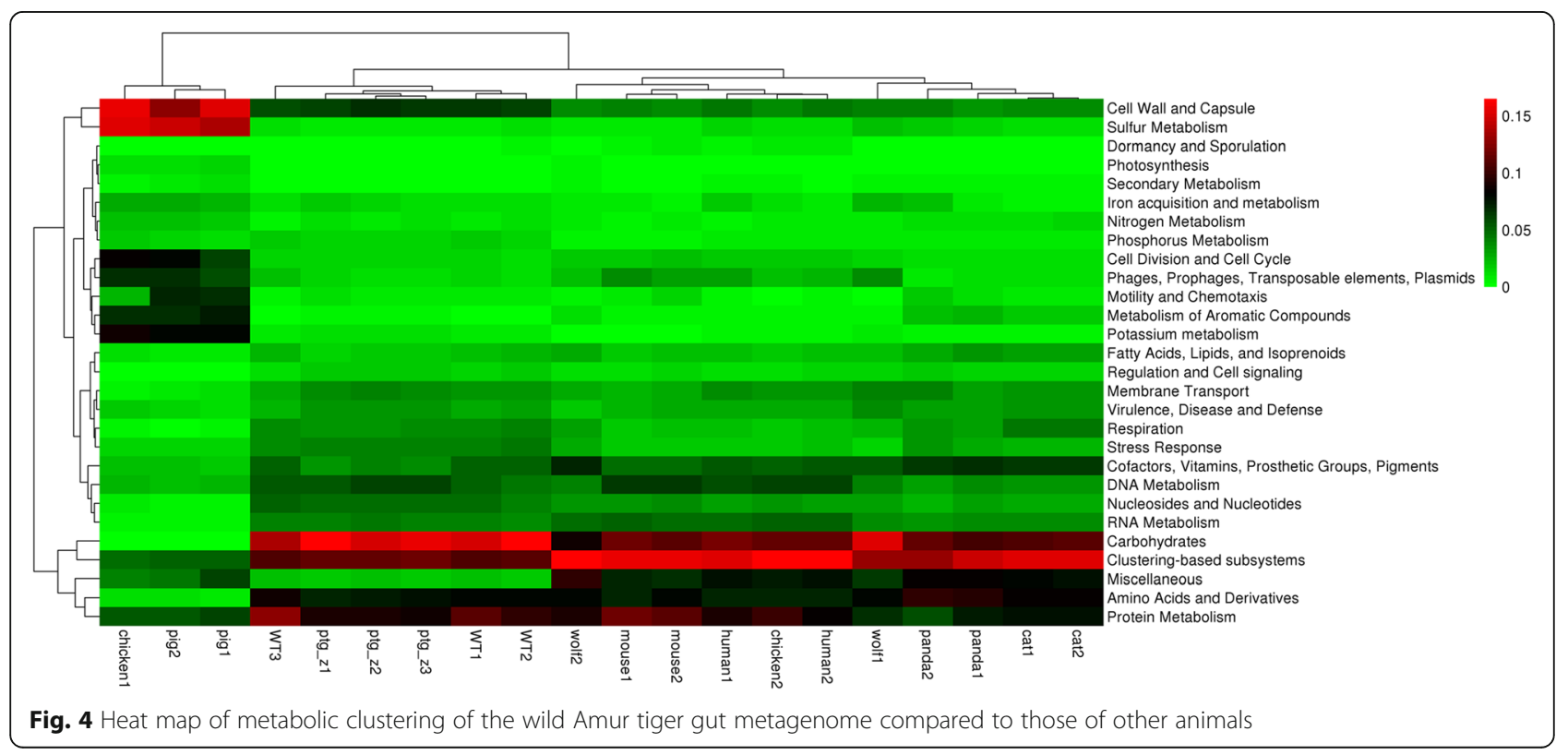


that the captive Amur tiger fecal metagenome had a lower abundance of Spirochaetes, Cyanobacteria and Ascomycota than other animals, and the primary functional categories were primarily associated with carbohydrate metabolism subsystems, clustering-based subsystems and protein metabolism. Taken together, these findings reveal the composition and functional structures of the gut microbiota in captive Amur tiger.

\section{Methods}

\section{Faecal sample collection}

Fresh faecal samples from 3 Amur tigers were collected at a single time point from the Heilongjiang Siberian Tiger Park in Heilongjiang Province, China, with permission form the authorities of the Heilongjiang Siberian Tiger Park. The age, sex and diet fed in the 10 days prior to fecal sampling are shown in Table 1 and 2. We randomly selected healthy Amur tigers in the Siberian Tiger Park, and faecal samples were collected aseptically immediately after defecation. The fresh faecal samples were transported to the laboratory on dry ice within $24 \mathrm{~h}$ of collection and stored at $-80^{\circ} \mathrm{C}$ until DNA extraction. We brought no toxic substance that would interfere with the animal habitats. The research complied with the protocols established by the China Wildlife Conservation Association and the legal requirements of China.

\section{DNA extraction}

DNA was extracted from the faecal samples using the E.Z.N.A. ' Stool DNA Kit (D4015-02, Omega, Inc., USA) according to the manufacturer's instructions. This reagent, which is designed to recover DNA from trace amounts of sample, has been shown to be effective for the preparation of DNA from most bacteria. Sample blanks consisted of unused swabs processed by DNA extraction and verified to contain no DNA amplicons. The total DNA was eluted in $50 \mu \mathrm{l}$ of Elution buffer by modification of the procedure described by the manufacturer (QIAGEN) and stored at $-80^{\circ} \mathrm{C}$ until measurement by PCR by LC-BIO TECHNOLOGIES (HANGZHOU) CO., LTD., Hang Zhou, Zhejiang Province, China.

\section{DNA library construction}

The DNA library was constructed using a TruSeq Nano DNA LT Library Preparation Kit (FC-121-4001). DNA was fragmented by dsDNA Fragmentase (NEB, M0348S)

Table 1 Sex, Age and Physical condition of captive Amur tiger in Siberian Tiger Park

\begin{tabular}{llll}
\hline Sample & Sex & Age (year) & Physical condition \\
\hline ptg_z1 & Male & 7 & Healthy \\
ptg_z2 & Male & 7 & Healthy \\
ptg_z3 & Female & 12 & Healthy \\
\hline
\end{tabular}

with incubation at $37^{\circ} \mathrm{C}$ for $30 \mathrm{~min}$. Library construction began with fragmented cDNA. Blunt-end DNA fragments were generated using a combination of fill-in reactions and exonuclease activity, and size selection was performed with the provided sample purification beads. An A-base was then added to the blunt ends of each strand to prepare them for ligation to the indexed adapters. Each adapter contained a T-base overhang to ligate the adapter to the A-tailed fragmented DNA. These adapters contained the full complement of the sequencing primer hybridization sites for the single, paired-end, indexed reads. Single- or dual-index adapters were ligated to the fragments, and the ligated products were amplified by PCR under the following conditions: initial denaturation at $95^{\circ} \mathrm{C}$ for $3 \mathrm{~min}$; 8 cycles of denaturation at $98^{\circ} \mathrm{C}$ for $15 \mathrm{~s}$, annealing at $60^{\circ} \mathrm{C}$ for $15 \mathrm{~s}$, and extension at $72{ }^{\circ} \mathrm{C}$ for $30 \mathrm{~s}$; and final extension at 37 ${ }^{\circ} \mathrm{C}$ for $5 \mathrm{~min}$.

\section{Data analysis}

Raw sequencing reads were processed to obtain valid reads for further analysis. First, sequencing adapters were removed from the sequencing reads using cutadapt v1.9. Second, low-quality reads were trimmed by fqtrim v0.94 using a sliding-window algorithm. Third, the reads were aligned to the host genome using bowtie 2 v2.2.0 to remove host contamination. The quality-filtered reads were then de novo assembled to construct the metagenome for each sample by SPAdes v3.10.0. All coding regions (CDS) of the metagenomic contigs were predicted by MetaGeneMark v3.26.

The CDS sequences of all samples were clustered by CD-HIT v4.6.1 to obtain unigenes. The unigene abundance for a certain sample was estimated by TPM based on the number of aligned reads by bowtie 2 v2.2.0. The lowest common ancestor taxonomy of the unigenes was obtained by aligning them against the NCBI NR database using DIAMOND v 0.7.12. Similarly, the functional annotation (GO, KEGG, CAZy) of the unigenes was obtained. Based on the taxonomic and functional annotations and abundance profiles of the unigenes, differential analysis was performed at the taxonomic, functional or gene level using Fisher's exact test (non-replicated groups) or the Kruskal-Wallis test (replicated groups).

\section{Comparative metagenomic analysis}

Comparative metagenomic analysis was performed using the MG-RAST pipelines. The metagenomic runs from the captive Amur tiger data were compared with the current publicly available gut metagenomes. In the MG-RAST metagenomic annotation pipeline, the captive Amur tiger fecal metagenomic datasets were compared with those of wild Amur tigers (WT1, WT2, and WT3) and fourteen public datasets from other animals, including cat 1 (mgm 
Table 2 The diet of captive Amur tiger in the 10 days before the sampling in Siberian Tiger Park

\begin{tabular}{|c|c|c|c|c|c|c|}
\hline \multirow[t]{2}{*}{ Date } & \multicolumn{2}{|l|}{ ptg_z1 } & \multicolumn{2}{|l|}{ ptg_z2 } & \multicolumn{2}{|l|}{ ptg_z3 } \\
\hline & Diet & $\begin{array}{l}\text { Quantity } \\
\text { (kg) }\end{array}$ & Diet & $\begin{array}{l}\text { Quantity } \\
(\mathrm{kg})\end{array}$ & Diet & $\begin{array}{l}\text { Quantity } \\
(\mathrm{kg})\end{array}$ \\
\hline 2017-6-10 & Fasting & - & Fasting & - & Fasting & - \\
\hline 2017-6-11 & Duck & 3.5 & Duck & 3.5 & Duck & 3.5 \\
\hline 2017-6-12 & Duck & 3.5 & Duck & 3.5 & Duck & 3.5 \\
\hline 2017-6-13 & Duck & 3.5 & Duck & 3.5 & Duck & 3.5 \\
\hline 2017-6-14 & Fasting & - & Fasting & - & Fasting & - \\
\hline 2017-6-15 & Duck & 3.5 & Duck & 3.5 & Duck & 3.5 \\
\hline 2017-6-16 & Duck & 3.5 & Duck & 3.5 & Duck & 3.5 \\
\hline 2017-6-17 & Fasting & - & Fasting & - & Fasting & - \\
\hline 2017-6-18 & Duck & 3.5 & Duck & 3.5 & Duck & 3.5 \\
\hline 2017-6-19 & Duck & 3.5 & Duck & 3.5 & Duck & 3.5 \\
\hline
\end{tabular}

4,626,753.3), cat 2 (mgm4626754.3), chicken 1 (mgm4743765.3), chicken 2 (mgm4440284.3), human 1 (mgm4472483.3), human 2 (mgm4440941.3), mouse 1 (mgm4537490.3), mouse 2 (mgm4540251.3), panda 1 (mgm4694746.3), panda 2 (mgm4683980.3), pig 1 (mgm 4745745.3), pig 2 (mgm4745743.3), wolf 1 (mgm45356 26.3) and wolf 2 (mgm4441601.3).

Integrate phylum level abundance information of captive Amur tiger and other species, standardize as a percentage, take the 25 phylum with the highest percentage, others are unclassified, take the $\log 10$ for heat map drawing (the value with a percentage of 0 is replaced by $1 / 2$ of the minimum value in all data), heat map drawing using the pheatmap package. Download the subsystems database on the SEED website (http://www.theseed.org) and comparison to unigenes of captive Amur tiger. Next, calculate the abundance of each category of subsystems, integrate abundance information of Amur tigers and other species subsystems, standardized as a percentage, using the pheatmap package to draw the heat map.

\section{Additional files}

Additional file 1: Information regarding the sequence data. (PDF $11 \mathrm{~kb}$ ) Additional file 2: Phylogenetic classification of the bacteria in the Amur tiger metagenome. (DOCX $537 \mathrm{~kb}$ )

Additional file 3: Phylogenetic classification of Eukaryota in the Amur tiger metagenome. (DOCX $22 \mathrm{~kb}$ )

Additional file 4: Phylogenetic classification of Archaea in the Amur tiger metagenome. (DOCX $21 \mathrm{~kb}$ )

Additional file 5: Phylogenetic classification of the viruses in the Amur tiger metagenome. (DOCX 43 kb)

Additional file 6: $\mathrm{GO}$ annotations of the Amur tiger faecal metagenome. (DOCX 683 kb)

Additional file 7: $\mathrm{GO}$ annotations of the Amur tiger faecal metagenome. (TIF $9899 \mathrm{~kb}$ )
Additional file 8: CAZy annotations of the Amur tiger metagenome. (DOCX $48 \mathrm{~kb}$ )

Additional file 9: CAZy classification of the Amur tiger faecal metagenome. (TIF $9289 \mathrm{~kb}$ )

\begin{abstract}
Abbreviations
CAZy: Carbohydrate-active enzymes; CBM: Carbohydratebinding modules; CITES: Convention on International Trade in Endangered Species of Wild Fauna and Flora; DNA: Deoxyribonucleic acid; GH: Glycoside hydrolase; GO: Gene ontology; GT: Glycosyl transferase; IUCN: International union for conservation of nature; KEGG: Kyoto encyclopedia of genes and genomes; NCBI: National center for biotechnology information; SRA: Short read archive
\end{abstract}

Acknowledgements

We thank Haitao Xu, Yurong Guo and Xuanmin Kong for sample collection.

Funding

This work was funded by the National Natural Science Foundation of China (31372209). This study also supported by "The Fundamental Research Funds for the Central Universities" (2572017AA20).

Availability of data and materials

All data are included in this article and its section "Additional files". The metagenomic sequence data from this study have been submitted to the National Center for Biotechnology Information (NCBI) Sequence Read Archive (SRA) under accession numbers SRP119699.

\section{Authors' contributions}

FH participated with the study design, bioinformatic analyses and manuscript preparation. LZ carried out sample collection and sample processing. JZ participated with statistical analyses. YM participated with bioinformatic analyses. DL provided faecal materials and back ground information of tigers for the study, and monitored the diet of the captive tigers before sample collection. YX contributed with bioinformatic and statistical analyses. GJ contributed analysis tools for the study and participated with bioinformatic analyses. KR and JM collaborated in the design and coordination and helped to draft the manuscript. All authors read and approved the final manuscript.

\section{Ethics approval}

The research complied with the protocols established by the China Wildlife Conservation Association and the legal requirements of China.

\section{Consent for publication}

Not applicable.

\section{Competing interests}

The authors declare that they have no competing interests.

\section{Publisher's Note}

Springer Nature remains neutral with regard to jurisdictional claims in published maps and institutional affiliations.

\section{Author details}

${ }^{1}$ College of Wildlife Resources, Northeast Forestry University, Harbin 150040, China. ${ }^{2}$ Heilongjiang Siberian Tiger Park, Harbin 150040, China. ${ }^{3}$ State Forestry Administration Detecting Center of Wildlife, Harbin 150040, China. ${ }^{4}$ China Feline Research Center of Chinese State Forestry Administration, Harbin 150040, China.

Received: 4 May 2018 Accepted: 12 November 2018 Published online: 04 December 2018

\section{References}

1. Mazak J. Panthera tigris. Mamm Species. 1981;152:1-8.

2. Miquelle DG, Pikunov DG, Dunishenko YM, Aramilev W, Nikolaev IG, Abramov VK, Smirnov EN, Salkina GP, Seryodkin IV, Gaponov W. 2005 Amur Tiger Census. Cat News. 2007:46:14-5. 
3. Zhang $C$, Zhang M, Stott P. Does prey density limit Amur tiger Panthera tigris altaica recovery in northeastern China? Wildlife Biol. 2013;19(4):452-61.

4. Ripple WJ, Estes JA, Beschta RL, Wilmers CC, Ritchie EG, Hebblewhite M, Berger J, Elmhagen B, Letnic M, Nelson MP. Status and ecological effects of the World's largest carnivores. Science. 2014;343(6167):1241484.

5. Ley RE, Hamady M, Lozupone C, Turnbaugh PJ, Ramey RR, Bircher JS, Schlegel ML, Tucker TA, Schrenzel MD, Knight R. Evolution of mammals and their gut microbes. Science. 2008;320(5883):1647-51.

6. Clemente JC, Ursell LK, Parfrey LW, Knight R. The impact of the gut microbiota on human health: an integrative view. Cell. 2012;148(6):1258-70.

7. Kau AL, Ahern PP, Griffin NW, Goodman AL, Gordon Jl. Human nutrition, the gut microbiome and the immune system. Nature. 2011;474(7351):327-36.

8. Ley R. Microbial ecology: human gut microbes associated with obesity. Nature. 2006;444(7122):1022.

9. Qin J, Li R, Raes J, Arumugam M, Burgdorf KS, Manichanh C, Nielsen T, Pons N, Levenez F, Yamada T. A human gut microbial gene catalogue established by metagenomic sequencing. Nature. 2010; 464(7285):59-65.

10. David LA, Maurice CF, Carmody RN, Gootenberg DB, Button JE, Wolfe BE, Ling AV, Devlin AS, Varma Y, Fischbach MA. Diet rapidly and reproducibly alters the human gut microbiome. Nature. 2014:505(7484):559.

11. Vital M, Gao J, Rizzo M, Harrison T, Tiedje JM. Diet is a major factor governing the fecal butyrate-producing community structure across Mammalia, Aves and Reptilia. Isme J. 2015;9(4):832.

12. Wang J, Tang $H$, Zhang $C$, Zhao $Y$, Derrien M, Rocher E, Vanhylckama JV, Strissel K, Zhao L, Obin M. Modulation of gut microbiota during probioticmediated attenuation of metabolic syndrome in high fat diet-fed mice. Isme J Multidiscipli. 2015;9(1):1.

13. Goodwin S, McPherson JD, McCombie WR. Coming of age: ten years of next-generation sequencing technologies. Nat Rev Genet. 2016;17(6):333.

14. Gill SR, Pop M, Deboy RT, Eckburg PB, Turnbaugh PJ, Samuel BS, Gordon II, Relman DA, Fraserliggett CM, Nelson KE. Metagenomic analysis of the human distal gut microbiome. Science. 2006;312(5778):1355-9.

15. Turnbaugh PJ, Hamady M, Yatsunenko T, Cantarel BL, Duncan A, Ley RE, Sogin ML, Jones WJ, Roe BA, Affourtit JP. A core gut microbiome in obese and lean twins. Nature. 2009;457(7228):480-4.

16. Warnecke $F$, Luginbühl $P$, Ivanova $N$, Ghassemian M, Richardson $T H$, Stege JT, Cayouette M, McHardy AC, Djordjevic G, Aboushadi N. Metagenomic and functional analysis of hindgut microbiota of a wood-feeding higher termite. Nature. 2007:450(7169):560-5.

17. Hess M, Sczyrba A, Egan R, Kim TW, Chokhawala H, Schroth G, Luo S, Clark DS, Chen F, Zhang T. Metagenomic discovery of biomass-degrading genes and genomes from cow rumen. Science. 2011;331(6016):463.

18. Lamendella R, Domingo JWS, Ghosh S, Martinson J, Oerther DB.

Comparative fecal metagenomics unveils unique functional capacity of the swine gut. BMC Microbiol. 2011;11(1):1-17.

19. Singh KM, Ahir VB, Tripathi AK, Ramani UV, Sajnani M, Koringa PG, Jakhesara S, Pandya PR, Rank DN, Murty DS. Metagenomic analysis of Surti buffalo (Bubalus bubalis) rumen: a preliminary study. Mol Biol Rep. 2012;39(4):4841.

20. Li RW, Connor EE, Li C, Baldwin Vi RL, Sparks ME. Characterization of the rumen microbiota of pre-ruminant calves using metagenomic tools. Environ Microbiol. 2012;14(1):129.

21. Lu HP, Wang YB, Huang SW, Lin CY, Wu M, Chih-Hao H, Yu HT. Metagenomic analysis reveals a functional signature for biomass degradation by cecal microbiota in the leaf-eating flying squirrel (Petaurista alborufus lena). BMC Genomics. 2012;13(1):466.

22. Bo X, Xu W, Li J, Dai L, Xiong C, Tang X, Yang Y, Mu Y, Zhou J, Ding J. Metagenomic analysis of the Rhinopithecus bieti fecal microbiome reveals a broad diversity of bacterial and glycoside hydrolase profiles related to lignocellulose degradation. BMC Genomics. 2015;16(1):174.

23. Zhu L, Wu Q, Deng C, Zhang M, Zhang C, Chen H, Lu G, Wei F. Adaptive evolution to a high purine and fat diet of carnivorans revealed by gut microbiomes and host genomes. Environ Microbiol. 2018;20(5):1711-22.

24. Roggenbuck M, Bærholm Schnell I, Blom N, Bælum J, Bertelsen MF, Sicheritz-Pontén T, Sørensen SJ, Gilbert MT, Graves GR, Hansen LH. The microbiome of New World vultures. Nat Commun. 2014;5:5498.

25. Keenan SW, Engel AS, Elsey RM. The alligator gut microbiome and implications for archosaur symbioses. Sci Rep. 2013;3:2877.

26. Spor A, Koren O, Ley R. Unravelling the effects of the environment and host genotype on the gut microbiome. Nat Rev Microbiol. 2011 9(4):279-90.
27. Larsson MHMA, Flores AS, Fedullo JDL, Mirandola RMS, Ito FH, Pessoa RB, Itikawa PH. Biochemical parameters of wild felids (Panthera leo and Panthera tigris altaica) kept in captivity. Semin Cienc Agrar. 2017;38(2):791.

28. Shrivatav AB, Singh KP, Mittal SK, Malik PK. Haematological and biochemical studies in tigers (Panthera tigris tigris). Eur J Wildl Res. 2012;58(1):365-7.

29. Junginger J, Hansmann F, Herder V, Lehmbecker A, Peters $M$, Beyerbach $M$, Wohlsein P, Baumgärtner W. Pathology in captive wild felids at German zoological gardens. PLoS One. 2015;10(6):e0130573.

30. Mohapatra RK, Panda S, Acharya UR. Study on activity pattern and incidence of stereotypic behavior in captive tigers. J Vet Behav. 2014;9(4):172-6.

31. Bashaw MJ, Kelling AS, Bloomsmith MA, Maple TL. Environmental effects on the behavior of zoo-housed lions and tigers, with a case study of the effects of a visual barrier on pacing. J Appl Anim Welf Sci. 2007;10(2):95-109.
Ready to submit your research? Choose BMC and benefit from:

- fast, convenient online submission

- thorough peer review by experienced researchers in your field

- rapid publication on acceptance

- support for research data, including large and complex data types

- gold Open Access which fosters wider collaboration and increased citations

- maximum visibility for your research: over $100 \mathrm{M}$ website views per year

At BMC, research is always in progress.

Learn more biomedcentral.com/submissions 